\title{
Shock waves simulated using the dual domain material point method combined with molecular dynamics
}

\author{
Duan Z. Zhang and Tilak R. Dhakal \\ Theoretical Division, Fluid Dynamics and Solid Mechanics Group \\ T-3, B216 \\ Los Alamos National Laboratory \\ Los Alamos, NM 87545, USA
}

January 2, 2017

\begin{abstract}
In this work we combine the dual domain material point method with molecular dynamics in an attempt to create a multiscale numerical method to simulate materials undergoing large deformations with high strain rates. In these types of problems, the material is often in a thermodynamically nonequilibrium state, and conventional constitutive relations or equations of state are often not available. In this method, the closure quantities, such as stress, at each material point are calculated from a molecular dynamics simulation of a group of atoms surrounding the material point. Rather than restricting the multiscale simulation in a small spatial region, such as phase interfaces, or crack tips, this multiscale method can be used to consider nonequilibrium thermodynamic effects in a macroscopic domain.

This method takes the advantage that the material points only communicate with mesh nodes, not among themselves; therefore molecular dynamics simulations for material points can be performed independently in parallel. The dual domain material point method is chosen for this multiscale method because it can be used in history dependent problems with large deformation without generating numerical noise as material points move across cells, and also because of its convergence and conservation properties.
\end{abstract}


To demonstrate the feasibility and accuracy of this method, we compare the results of a shock wave propagation in a cerium crystal calculated using the direct molecular dynamics simulation with the results from this combined multiscale calculation.

\section{Introduction}

The equation of state or constitutive relation of a material is often obtained by assuming thermodynamic equilibrium. As a consequence of this assumption, the equation of state can only be applied to problems in which the time scale of the material deformation, defined by the inverse of the characteristic strain rate, is significantly larger than the time scale for the molecules in the material to relax to their thermodynamic equilibrium state after an external perturbation. For problems with a smaller ratio between the problem time scale and the relaxation time scale of the material, the equation of state cannot be used. These types of physical problems are the main focus of the present paper.

Since the time scale of the material deformation of concern here is comparable to or even smaller than the thermodynamic relaxation time, the material response at a given external disturbance depends on the microstructures of the material at that time, which depends on the history of the material. Therefore, the material behavior is generally history dependent for these types of problems. Furthermore, such high rates of material deformation often involve significant forces, for instance in hypervelocity impacts. Significant material deformations are common in these cases.

To numerically simulate these types of problems at the macroscopic level, on the theoretical front, one needs a set of continuum scale equations with a method to calculate the closure quantities accurately representing the microstructure and history effects. On the numerical front, one needs a numerical method capable of accurately tracking material deformation history in cases of large material deformation.

The continuum scale equations can be derived from the Liouville equation using ensemble average for fairly general cases $[1,2]$. The closure quantities, such as pressure, stress, and heat flux, are directly related to microscopic interactions among molecules and atoms. In this paper, we use these relations to calculate the necessary closure quantities directly from molecular dynamics (MD) simulations. Such closure quantities are used in continuum calculations to advance the 
macroscopic quantities. For thermodynamically equilibrium systems in the limit of Continuum Mechanics, we show that such calculated stress is consistent with the traditional concept. The method presented in the current paper is in the same philosophy of the heterogeneous multiscale method (HMM) [3, 4, 5, 6, 7]. For selected regions in a macroscopic domain, lower length scale simulations are performed in synchrony with the macroscopic continuum level calculation. These lower length scale simulations are constrained by the macroscopic information passed to them. Closure quantities, such as stress or heat flux, calculated from these lower length scale systems are passed to the continuum level calculation to advance the macroscopic state. In this work we emphasize the simulation method for history dependent systems undergoing large macroscopic deformations. Frequent reinitialization and time averaging are not allowed. While the non-linear terms are neglected in $[3,5]$ and the work is limited to small material deformation, the Lagrangian coordinate system is capable of tracking material deformation history, which is an advantage of a Lagrangian method. The main disadvantage of a pure Lagrangian method, such as the Lagrangian finite element method, appears in problems involving large material deformation, where elements are often severely distorted and even entangled leading to loss of accuracy and eventual failure of the calculation. In the present paper, we use the dual domain material point (DDMP) method to avoid this numerical difficulty. For the fluid dynamics problems studied in [4], the simulated molecular dynamics systems are fixed in the spatial locations intended for history independent materials, such as Newtonian fluids, based on the pure Eulerian description. Unless special treatments, such as in $[8,9]$, are used, Eulerian methods often lead to significant numerical diffusion of history dependent variables. Rather than proposing another multiscale computation philosophy or a method to perform MD simulations, the main purpose of this work is to show that the general strategy of HMM can be efficiently implemented with the dual domain material point (DDMP) method for thermodynamically nonequilibrium or history dependent systems undergoing large material deformation by taking advantage of the simultaneous use of Lagrangian particles and Eulerian meshes in the method.

The Lagrangian particles, also called material points, are used to track the history of the material deformation. These particles can be regarded as pieces of the material experiencing the history. The Eulerian mesh is used to perform numerical analysis, such as taking spatial derivatives. Since the information about material history is carried by the Lagrangian particles, numerical diffusion 
for the history dependent quantities is avoided. Furthermore, because the mesh is Eulerian, it remains unchanged in cases of large material deformations, thereby avoiding the mesh distortion difficulty suffered by a pure Lagrangian method. In the DDMP method, the closure quantities, such as the stress, are needed only at material points following the motion of the material. The numerical method does not require direct molecular dynamics simulation of the entire macroscopic computational domain. Direct molecular dynamics simulations are only performed in small representative domains around the material points. These simulations are driven by the continuum level DDMP calculation by imposing appropriate constraints to the molecular dynamics systems. The closure quantities calculated from these molecular systems are used to drive the macroscopic DDMP calculation. Such intimate communications between the macroscopic scale calculation and the microscopic scale simulations are unique for these types of multiscale calculations and need to be studied carefully to ensure physical consistency of the results.

In the DDMP method, material points only communicate with mesh nodes and not directly among themselves. Therefore, there is no need to form neighbor lists of the particles. This feature of the numerical method is very advantageous for parallel computation, especially for heterogeneous computers, such as CPU-GPU combined platforms. In the present paper, we take advantage of this feature to demonstrate the feasibility of such multiscale calculations.

\section{Continuum scale equations and their solution method}

\subsection{Derivation of continuum scale equations}

For a generic quantity $g_{\alpha}$ pertaining to atom $\alpha$, the corresponding average $\bar{g}$ at time $t$ and position $\boldsymbol{x}$ is defined using

$$
n(\boldsymbol{x}, t) \bar{g}(\boldsymbol{x}, t)=\int \sum_{\alpha=1}^{N} \delta\left(\boldsymbol{x}_{\alpha}(t)-\boldsymbol{x}\right) g_{\alpha}(\mathcal{C}, t) P(\mathcal{C}, t) d \mathcal{C},
$$

where $n$ is the number density of the atoms which corresponds to $g_{\alpha}=\bar{g}=1$ in the above equation, $N$ is the number of atoms in the system, $\mathcal{C}=\left\{\boldsymbol{x}_{1}, \cdots, \boldsymbol{x}_{N} ; \boldsymbol{v}_{1}, \cdots, \boldsymbol{v}_{N}\right\}$ is a point in the positionvelocity phase space for the system, and $P(\mathcal{C}, t)$ is the classical $N$-atom probability density function in the phase space. The evolution of this probability density function is described by the Liouville 
equation,

$$
\frac{\partial P}{\partial t}+\sum_{j=1}^{N} \nabla_{\boldsymbol{x}_{j}} \cdot\left(\boldsymbol{v}_{j} P\right)+\sum_{j=1}^{N} \nabla \boldsymbol{v}_{j} \cdot\left(\dot{\boldsymbol{v}}_{j} P\right)=0
$$

By multiplying $\delta\left(\boldsymbol{x}_{\alpha}-\boldsymbol{x}\right) g_{\alpha}$ through the equation and summing over all atoms in the system, and then integrating over all possible configurations $(\mathcal{C}$ 's), one finds the continuum level transport equation for the average quantity $\bar{g}[1,2]$,

$$
\frac{\partial(n \bar{g})}{\partial t}+\nabla \cdot(n \bar{g} \boldsymbol{v})=n \frac{\overline{d g}}{d t} .
$$

Let $g=m$ and mass density $\rho=n \bar{m}$. We find the mass conservation equation

$$
\frac{\partial \rho}{\partial t}+\nabla \cdot(\rho \widetilde{\boldsymbol{v}})=0
$$

where $\widetilde{\boldsymbol{v}}=n \overline{m \boldsymbol{v}} / \rho$ is the Favre average velocity.

Letting $g=m \boldsymbol{v}$, one finds the momentum equation

$$
\frac{\partial \rho \widetilde{\boldsymbol{v}}}{\partial t}+\nabla \cdot(\rho \widetilde{\boldsymbol{v}} \widetilde{\boldsymbol{v}})=\nabla \cdot \boldsymbol{\sigma}_{k}+n \overline{\boldsymbol{f}}+\rho \boldsymbol{b}
$$

where $\boldsymbol{b}$ is the body force, $\boldsymbol{\sigma}_{k}$ is the stress due to velocity fluctuations,

$$
\boldsymbol{\sigma}_{k}(\boldsymbol{x}, t)=-\int \sum_{\alpha=1}^{N} \delta\left(\boldsymbol{x}_{\alpha}-\boldsymbol{x}\right) m_{\alpha}\left(\boldsymbol{v}_{\alpha}-\widetilde{\boldsymbol{v}}\right)\left(\boldsymbol{v}_{\alpha}-\widetilde{\boldsymbol{v}}\right) P(\mathcal{C}, t) d \mathcal{C},
$$

and

$$
n \overline{\boldsymbol{f}}=\int \sum_{\alpha=1}^{N} \delta\left(\boldsymbol{x}_{\alpha}-\boldsymbol{x}\right) \boldsymbol{f}_{\alpha}(\mathcal{C}, t) P(\mathcal{C}, t) d \mathcal{C},
$$

with $\boldsymbol{f}_{\alpha}(\mathcal{C}, t)$ being the total force acting on atom $\alpha$ by other atoms in configuration $\mathcal{C}$. Let $\boldsymbol{f}_{\alpha \beta}$ be the force atom $\beta$ acting on $\alpha$. The total force can be written as

$$
\boldsymbol{f}_{\alpha}=\sum_{\beta=1}^{N} \boldsymbol{f}_{\alpha \beta}
$$

Using the Taylor expansion of the $\delta$-function in $(7)$, it is shown $[1,10]$ that

$$
n \overline{\boldsymbol{f}}=\nabla \cdot \boldsymbol{\sigma}_{f},
$$

where

$$
\boldsymbol{\sigma}_{f}(\boldsymbol{x}, t)=\frac{1}{2} \int \sum_{\alpha=1}^{N} \sum_{\beta=1}^{N} \delta\left(\xi \boldsymbol{x}_{\beta}+(1-\xi) \boldsymbol{x}_{\alpha}-\boldsymbol{x}\right)\left(\boldsymbol{x}_{\beta}-\boldsymbol{x}_{\alpha}\right) \boldsymbol{f}_{\alpha \beta}(\mathcal{C}, t) P(\mathcal{C}, t) d \mathcal{C},
$$


is the stress due to interaction forces among the atoms with $\xi$ being a coefficient between $[0,1]$ depending on configuration $\mathcal{C}$ and time $t$.

Using (9), we can write the momentum equation (5) in terms of the total Cauchy stress $\boldsymbol{\sigma}=$ $\boldsymbol{\sigma}_{k}+\boldsymbol{\sigma}_{f}$

$$
\frac{\partial \rho \boldsymbol{v}}{\partial t}+\nabla \cdot(\rho \widetilde{\boldsymbol{v}} \widetilde{\boldsymbol{v}})=\nabla \cdot \boldsymbol{\sigma}+\rho \boldsymbol{b}
$$

Although the derivation of stress in $[1,2]$ uses the abstract Taylor expansion of the $\delta$-function, it is equivalent to translating configuration $\mathcal{C}$ by a small distance of order of atomic length scale $\ell_{a}$ in the physical space $[10,11]$, assuming the probability distribution function $P(\mathcal{C}, t)$ is differentiable with respect to this translation. This assumption implies separation between the atomic length scale $\ell_{a}$ and the macroscopic length scale $L_{m}\left(\ell_{a}<<L_{m}\right)$. Under this assumption, within an error of order $\left(\ell_{a} / L_{m}\right)^{2}$ in momentum equation (11), we can set $\xi=0$ in (10).

The stress $\boldsymbol{\sigma}_{f}$ defined in (10) is not the only one satisfying (9). Stress $\boldsymbol{\sigma}_{f}$ can differ by a divergence-free tensor without affecting the dynamics of the system. Furthermore, for a given potential among atoms, the stress defined in (10) is still not unique, because there are different ways $[12,13]$ of decomposing the force acting on an atom into pair interaction forces while yielding the same total force in (8). Different decompositions lead to different $\boldsymbol{f}_{\alpha \beta}$, and different stresses calculated from (10). In continuum mechanics, the difference in the divergence-free stress is often regarded as a residual stress, which depends on the definition of the "undeformed", or the reference state.

In principle, this non-uniqueness of stress does not prohibit us to perform the multiscale calculation described in the present paper, because it is the divergence of the stress, not the stress itself, drives the evolution of the macroscopic momentum as in (11). However, it is still desirable, at least comforting, to know that this stress is consistent with the stress defined in continuum mechanics at the limit of thermodynamic equilibrium.

Let $\boldsymbol{x}$ be the geometric center of a representative volume $V_{c}$ with length scale $L_{c}\left(>>\ell_{a}\right)$. If $L_{c}<<L_{m}$, we have

$$
\boldsymbol{\sigma}(\boldsymbol{x}, t)=\overline{\boldsymbol{\sigma}}^{v}(\boldsymbol{x}, t)+O\left(\frac{L_{c}^{2}}{L_{m}^{2}}\right)=\overline{\boldsymbol{\sigma}}_{k}^{v}(\boldsymbol{x}, t)+\overline{\boldsymbol{\sigma}}_{f}^{v}(\boldsymbol{x}, t)+O\left(\frac{L_{c}^{2}}{L_{m}^{2}}\right),
$$

where over bar with superscript $v,\left(\leftarrow^{v}\right)$, denotes average over the representative volume $V_{c}$. Using (10) after exchanging the order of the volume and ensemble averages, (the order of the probability 
integration with the volume integration) and setting $\xi=0$, we have

$$
\begin{aligned}
\overline{\boldsymbol{\sigma}}^{v}(\boldsymbol{x}, t) & =\frac{1}{V_{c}} \int_{V_{c}} \boldsymbol{\sigma}(\boldsymbol{y}, t) d^{3} y \\
& =\int \frac{1}{V_{c}} \sum_{\boldsymbol{x}_{\alpha} \in V_{c}}\left[-m_{\alpha}\left(\boldsymbol{v}_{\alpha}-\widetilde{\boldsymbol{v}}\right)\left(\boldsymbol{v}_{\alpha}-\widetilde{\boldsymbol{v}}\right)+\frac{1}{2} \sum_{\beta}\left(\boldsymbol{x}_{\beta}-\boldsymbol{x}_{\alpha}\right) \boldsymbol{f}_{\alpha \beta}\right] P(\mathcal{C}, t) d \mathcal{C}+O\left(\frac{\ell_{a}}{L_{m}}\right),
\end{aligned}
$$

where the first summation is over all the atoms in the representative volume $V_{c}$, and the second summation is over all the atoms interacting with atom $\alpha\left(\boldsymbol{f}_{\alpha \beta} \neq \mathbf{0}\right)$. If atom $\alpha$ is close to a boundary of $V_{c}$, atom $\beta$ could be outside the volume, but must be within the vicinity, (within a distance of order $\ell_{a}$ ), of the boundary, if the range of the interaction forces is of that order. Such cross-boundary interacting atom pairs are located in the region near the boundary. The volume of the boundary region is of order $\ell_{a} L_{c}^{2}$. Neglecting such pair interactions in the boundary region causes an error of order $\ell_{a} / L_{c}$ in the stress, which is insignificant for $L_{c}>>\ell_{a}$. Allowing this error, to calculate the volume averaged stress $\overline{\boldsymbol{\sigma}}^{v}$, we can only consider the interaction pairs inside $V_{c}$ and approximate $\sum_{\beta}$ in (13) with $\sum_{\boldsymbol{x}_{\beta} \in V_{c}}$. Noting $\boldsymbol{f}_{\alpha \beta}=-\boldsymbol{f}_{\beta \alpha}$, we have

$$
\sum_{\boldsymbol{x}_{\alpha} \in V_{c}} \sum_{\boldsymbol{x}_{\beta} \in V_{c}}\left(\boldsymbol{x}_{\beta}-\boldsymbol{x}_{\alpha}\right) \boldsymbol{f}_{\alpha \beta}=-\sum_{\boldsymbol{x}_{\beta} \in V_{c}} \boldsymbol{x}_{\beta} \sum_{\boldsymbol{x}_{\alpha} \in V_{c}} \boldsymbol{f}_{\beta \alpha}-\sum_{\boldsymbol{x}_{\alpha} \in V_{c}} \boldsymbol{x}_{\alpha} \sum_{\boldsymbol{x}_{\beta} \in V_{c}} \boldsymbol{f}_{\alpha \beta}=-2 \sum_{\boldsymbol{x}_{\alpha} \in V_{c}} \boldsymbol{x}_{\alpha} \boldsymbol{f}_{\alpha}^{i n t},
$$

where $\boldsymbol{f}_{\alpha}^{i n t}=\sum \boldsymbol{x}_{\beta} \in V_{c} \boldsymbol{f}_{\alpha \beta}$ is the sum of the forces from the atoms inside the representative volume, or the total internal force. Using (14) in (13), under condition $\ell_{a}<<L_{c}<<L_{m}$, we find

$$
\overline{\boldsymbol{\sigma}}^{v}=-\int \frac{1}{V_{c}} \sum_{\boldsymbol{x}_{\alpha} \in V_{c}}\left[m_{\alpha}\left(\boldsymbol{v}_{\alpha}-\widetilde{\boldsymbol{v}}\right)\left(\boldsymbol{v}_{\alpha}-\widetilde{\boldsymbol{v}}\right)+\boldsymbol{x}_{\alpha} \boldsymbol{f}_{\alpha}^{i n t}\right] P(\mathcal{C}, t) d \mathcal{C}+O\left(\frac{L_{c}^{2}}{L_{m}^{2}}\right)+O\left(\frac{\ell_{a}}{L_{c}}\right) .
$$

In almost all practical cases, when the representative volume is sufficiently large $\left(L_{c}>>\ell_{a}\right)$, the volume averaged value in (15) or (13) becomes insensitive to configuration $\mathcal{C}$. Noting that $\int P(\mathcal{C}, t) d \mathcal{C}=1$, using (12), (13) and (15), under condition $\ell_{a}<<L_{c}<<L_{m}$ we have

$$
\begin{aligned}
\boldsymbol{\sigma} \approx \overline{\boldsymbol{\sigma}}^{v} & \approx \frac{1}{V_{c}} \sum_{\boldsymbol{x}_{\alpha} \in V_{c}}\left[-m_{\alpha}\left(\boldsymbol{v}_{\alpha}-\widetilde{\boldsymbol{v}}\right)\left(\boldsymbol{v}_{\alpha}-\widetilde{\boldsymbol{v}}\right)+\frac{1}{2} \sum_{\beta}\left(\boldsymbol{x}_{\beta}-\boldsymbol{x}_{\alpha}\right) \boldsymbol{f}_{\alpha \beta}\right] \\
& =-\frac{1}{V_{c}} \sum_{\boldsymbol{x}_{\alpha} \in V_{c}}\left[m_{\alpha}\left(\boldsymbol{v}_{\alpha}-\widetilde{\boldsymbol{v}}\right)\left(\boldsymbol{v}_{\alpha}-\widetilde{\boldsymbol{v}}\right)+\boldsymbol{x}_{\alpha} \boldsymbol{f}_{\alpha}^{i n t}\right]+O\left(\frac{\ell_{a}}{L_{c}}\right) .
\end{aligned}
$$

In deriving these relations, we have only used the separation of the atomic and the macroscopic length scales. This length scale separation allows us to calculate the stress as an average over a 
representative volume with a length scale between the atomic and the macroscopic length scales without explicitly specifying the probability distribution function $P(\mathcal{C}, t)$.

The second line of (16) is the virial expression of the Cauchy stress. In continuum mechanics under the assumption of thermodynamic equilibrium, the expression can be obtained by differentiating the Helmholtz free energy with respect to the deformation gradient tensor [12, 14]. Therefore, the stress $\boldsymbol{\sigma}$ used in this work is consistent with the stress in classical continuum mechanics.

In our MD simulation, the representative volume is a periodic box described in the next section. To avoid the error related to interaction pairs near the boundaries in our computational domain and to take advantage of the periodic boundary conditions, the potential part of stress $\overline{\boldsymbol{\sigma}}^{v}$ is calculated using the first line of (16) with pair interaction forces. No assumption of thermodynamic equilibrium is necessary in this derivation and calculation.

\subsection{Solution method for the continuum equations}

In thermodynamic nonequilibrium systems, the stress is generally history dependent, even for simple inter-atomic interactions, such as gases. To numerically simulate these systems, one needs to deal with two significant challenges. The first one is to have an efficient method for the atomic scale physics that can calculate the stress in a state far away from thermodynamic equilibrium. The second one is the need for a continuum scale numerical method that can accurately track material deformation history under the condition of extreme material deformation. In this subsection, we briefly describe this continuum scale numerical method. The method addressing the first challenge will be discussed in the next section.

For the continuum scale calculation, we choose the dual domain material point (DDMP) method [15] enhanced with sub-points [16]. The DDMP method uses Lagrangian particles, also called material points, in addition to an Eulerian grid, where the computational cells and nodal points are fixed in space. The Lagrangian particles carry information of material deformation and naturally track the history of the material. Conservation quantities, such as mass and momentum are calculated on the Eulerian mesh nodes. The Lagrangian particles are seeded initially in the Eulerian grid. A typical seeding method is to divide an Eulerian cell into smaller regions and then to place material points in the centers of these regions. The initial volume of the particle is assigned to be the volume of the region. The subsequent particle volume is updated each time step according 
to the velocity divergence at the particle location. After initial seeding, these material points, or particles, are allowed to move following the motion of the material.

The method seeks an approximate weak solution to the momentum equation (11) similar to the finite element method. It uses the same shape functions for the given mesh as in the finite element method. The velocity at position $\boldsymbol{x}$ is approximated by interpolating the surrounding nodal velocities to the position as

$$
\widetilde{\boldsymbol{v}}(\boldsymbol{x}, t)=\sum_{i=1}^{N_{g}} \widetilde{\boldsymbol{v}}_{i}(t) S_{i}(\boldsymbol{x}),
$$

where $N_{g}$ is the number of grid points in the domain, $\widetilde{\boldsymbol{v}}_{i}$ is the nodal velocity, and $S_{i}(\boldsymbol{x})$ is the shape function associated with node $i$. By using the same shape functions to approximate the virtual velocity, $\delta \boldsymbol{v}=\sum_{i=1}^{N_{g}} \delta \boldsymbol{v}_{i} S_{i}(\boldsymbol{x})$, multiplying the virtual velocity to both sides of (11), then integrating over the computation domain, and noting that $\delta \boldsymbol{v}_{i}$ is arbitrary, one finds the discretized momentum equation [17]:

$$
m_{i} \frac{\Delta \widetilde{\boldsymbol{v}}_{i}}{(\Delta t)_{c m}}=\boldsymbol{f}_{i}^{i n t}+\int_{\partial \Omega_{f}} \boldsymbol{\sigma} \cdot \boldsymbol{n} S_{i} d A+m_{i} \boldsymbol{b}, \quad \boldsymbol{f}_{i}^{i n t}=-\int_{\Omega} \boldsymbol{\sigma} \cdot \nabla S_{i}(\boldsymbol{x}) d v \approx-\sum_{p=1}^{N_{p}} v_{p} \boldsymbol{\sigma}_{p} \cdot \nabla S_{i}\left(\boldsymbol{x}_{p}\right),
$$

where $(\Delta t)_{c m}$ the time step in this continuum macroscopic calculation, $m_{i}=\int_{\Omega} \rho S_{i} d v$ the mass associated with node $i$ on the Eulerian grid, $\boldsymbol{f}_{i}^{\text {int }}$ the DDMP internal force, $\boldsymbol{n}$ the outward normal on traction boundary $\partial \Omega_{f}, \sigma_{p}$ the stress at material point $p, v_{p}$ the volume of the material point, and $N_{p}$ the number of material points in the computational domain $\Omega$, which is chosen to contain large enough space for the material to move and deform. For the one-dimensional examples in the present paper, the boundary conditions at both ends are specified with $\widetilde{\boldsymbol{v}}=\mathbf{0}$, not traction boundary conditions, therefore the surface integral in the first equation of (18) vanishes.

In contrast to a finite element calculation, in material point methods, the computational domain can contain many empty cells. Although book keeping and computer memory are needed for these empty cells, the computational effort used for these cells is negligible [18], as the dominant computational cost in material point methods is updating state variables, volumes, and positions of the material points.

Equation (18) is used directly in the original MPM [17]. A significant problem arises due to the discontinuity in the shape function gradient, $\nabla S_{i}\left(\boldsymbol{x}_{p}\right)$, which is illustrated for a one-dimensional case in Fig. 1. The discontinuity of the gradient at node $i$ causes a particle contribution to the 


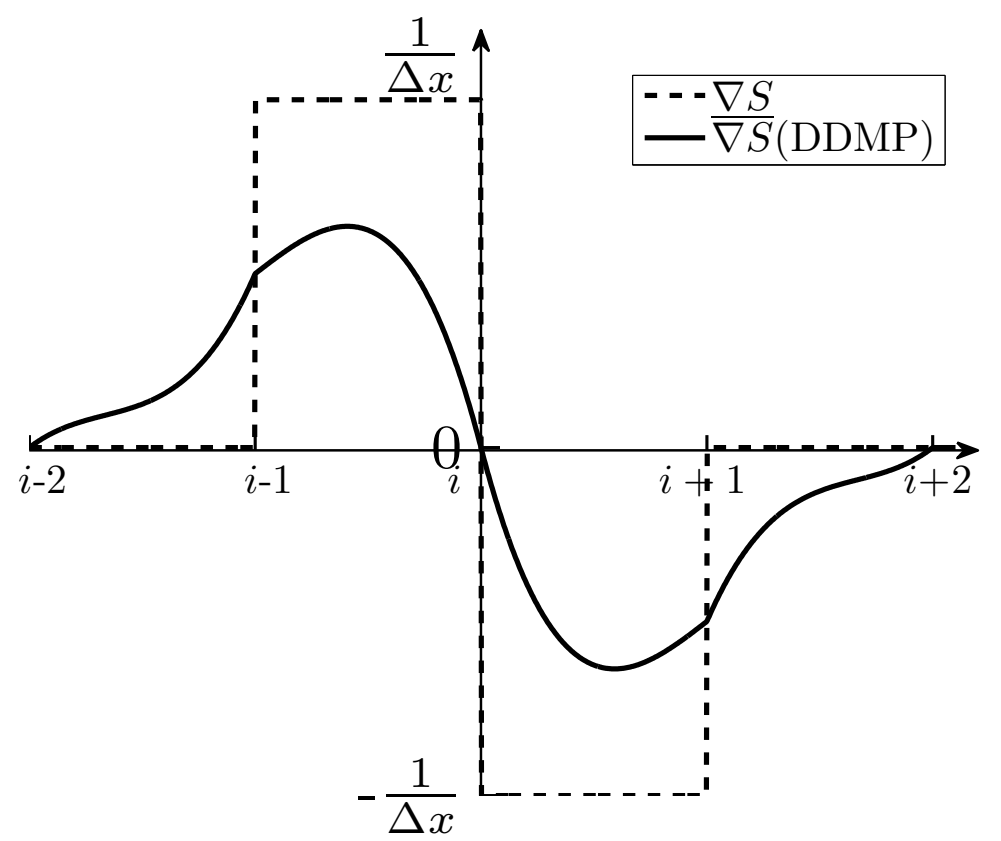

Figure 1: Illustration of the gradient of the shape function used in traditional MPM (dotted line) and used in DDMP (solid line) at node $i . \Delta x$ is the grid spacing.

internal force $\boldsymbol{f}_{i}^{\text {int }}$ in (18) to suddenly switch signs as the particle crosses a cell boundary, creating numerical noise that could lead to numerical instability.

To avoid this numerical noise, the DDMP method [15] uses identical shape functions as in the original MPM, but replaces the stress $\boldsymbol{\sigma}$ in (18) with a stress that differs from the original one by a quantity of order $(\Delta x / L)^{2}$, such that the volume integral in (18) can be calculated as if the gradient $\nabla S_{i}\left(\boldsymbol{x}_{p}\right)$ in (18) is modified to be

$$
\overline{\nabla S_{i}}\left(\boldsymbol{x}_{p}\right)=\alpha\left(\boldsymbol{x}_{p}\right) \nabla S_{i}\left(\boldsymbol{x}_{p}\right)+\left[1-\alpha\left(\boldsymbol{x}_{p}\right)\right] \widetilde{\nabla S_{i}}\left(\boldsymbol{x}_{p}\right),
$$

where

$$
\alpha\left(\boldsymbol{x}_{p}\right)=0.5\left\{\prod_{k=1}^{n_{c}}\left[2 S_{k}\left(\boldsymbol{x}_{p}\right)\right]\right\}^{\frac{3}{2\left(n_{c}-1\right) d}},
$$

and

$$
\widetilde{\nabla S_{i}}\left(\boldsymbol{x}_{p}\right)=\sum_{j=1}^{N} \frac{S_{j}\left(\boldsymbol{x}_{p}\right)}{V_{j}} \int_{\Omega} S_{j} \nabla S_{i} d v
$$

with $V_{j}$ being the control volume at node $j, n_{c}$ being the number of nodes in a computational cell, and $d$ being the number of spatial dimensions. This modified gradient of the shape function is also illustrated in Fig. 1 for a one-dimensional case. Such a modified gradient of the shape function is 
continuous, although not smooth. Since the modified gradient of the shape function has a larger support (nonzero region) than that of the shape function, this method is called the dual domain material point (DDMP) method. Although the support of the modified gradient of shape function is enlarged to include the immediate neighbor cells, all the non-local effects of a particle can be accounted for by two consecutive local operations. Therefore this scheme can be implemented in calculations with an unstructured mesh. The numerical noise caused by the discontinuity of $\nabla S_{i}$ is eliminated when $\overline{\nabla S_{i}}(\boldsymbol{x})$ replaces $\nabla S_{i}$. It is proven [15] that the modified gradient $\overline{\nabla S_{i}}(\boldsymbol{x})$ satisfies all the properties sufficient [19] for the method to converge to the solution of the partial differential equation as the mesh and the time step are refined. Furthermore, for a given mesh, the approximate solution guarantees mass and momentum conservation and has second-order accuracy in energy conservation with respect to spatial and time discretization.

Examples $[15,20,21,22]$ have shown that the use of DDMP eliminates the noise generated by cell crossing particles. After nodal accelerations are calculated from (18), the material point velocities are updated using nodal accelerations, which are interpolated from the nodal positions to the particle locations as described by the fluid-implicit-particle method (FLIP) [23]. Instead of directly interpolating the node velocities to particles, this scheme for particle velocity update ensures that the particle-in-cell (PIC) [24] family of methods (including MPM and DDMP) have very small (sometimes even regarded as zero) numerical diffusion. This is because the calculated particle velocity will not change unless the right hand side of (18) is nonzero. In other words, all the change in the particle velocity is caused by the physical driving force represented by the right hand side of (18), and not by any numerical effects.

The DDMP method described above can be used to compute large material deformation, provided the stresses at the material points can be obtained. In the present work, we study a method of performing macroscopic simulations for systems far away from their thermodynamic equilibriums by computing these stresses directly from molecular dynamics simulations at each material point. We take the advantage that the material points are Lagrangian points. These points are tracked throughout the computation so that the history of the material can be considered in the stress calculation. In these types of calculations, the stress evaluation at material points is expensive. To reduce the number of material points needed, while having sufficient numerical accuracy in the DDMP internal force calculation, the recently developed sub-point algorithm [16] is used. The 


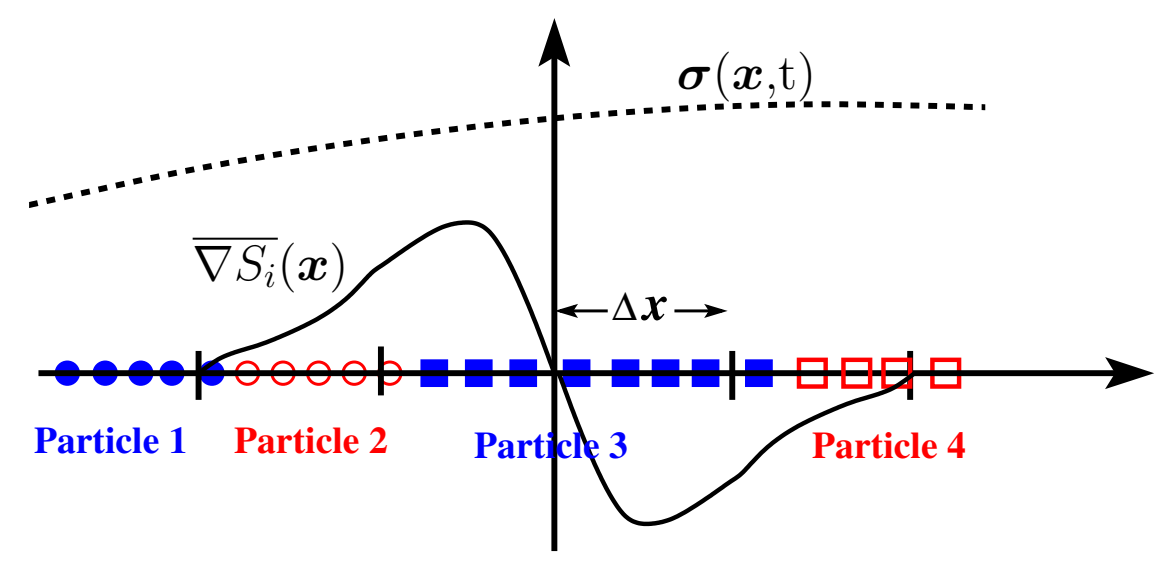

Figure 2: One-dimensional illustration of the sub-point algorithm. Four particles or material points are divided into 22 sub-points, represented by hollow or solid circles and squares. Each of these sub-points is an integration point in (23). The sub-point belonging to the same particle share the same value of the stress.

algorithm exploits the difference between the stress variation length scale, which is $L_{m}$, and the length scale of the shape function, which is the typical mesh size $\Delta x$. As illustrated in Fig. 2, in a meaningful calculation, the typical mesh size $\Delta x<<L_{m}$. The stress varies much slower than the modified gradient $\overline{\nabla S_{i}}$ of the shape function. To ensure numerical accuracy in the internal force calculation, we split a material point, or particle, into a number of integration points, called sub-points. Distribution of these sub-points can be very flexible [16]. For the one-dimensional cases studied in the present paper, we can easily track the domain of a particle and evenly distribute subpoints in the particle domain. For higher dimensional problems, the distribution of the sub-points is discussed in [16].

The values of $\overline{\nabla S_{i}}$ are evaluated individually on these sub-points to account for rapid variation of the modified gradient of the shape function, while the sub-points belonging to the same particle share the same stress. These sub-points can be generated at each time step around a particle. They are introduced purely to account for variations of $\overline{\nabla S_{i}}$ and do not carry any physical information; therefore there is no need to track the history of these sub-points. As in the ordinary material point methods, the history information is carried by the particles. The MD simulation to calculate stresses are performed for the particles, not for sub-points. Since the generation and calculation associated with these sub-points are very cheap compared to the stress calculation on the particles, 
a large number of sub-points can be used without incurring too much computational cost. As shown in [16], the numerical accuracy of this sub-point algorithm is very close to the DDMP calculation as if using the same number of particles instead of sub-points. This sub-point DDMP method is equivalent to further replacing the gradient of the shape function in (18) by

$$
\overline{\overline{\nabla S_{i}}}\left(\boldsymbol{x}_{p}\right)=\frac{1}{n_{p s}} \sum_{s=1}^{n_{p s}} \overline{\nabla S_{i}}\left(\boldsymbol{x}_{p s}\right),
$$

where $n_{p s}$ is the number of sub-points for material point $p$, and $\boldsymbol{x}_{p s}$ is the position of a sub-point, which is generated around the material point at each time step. In this way, the internal force in (18) is calculated by

$$
\boldsymbol{f}_{i}^{i n t}=-\int_{\Omega} \boldsymbol{\sigma} \cdot \nabla S_{i}(\boldsymbol{x}) d v \approx-\sum_{p=1}^{N_{p}} \frac{v_{p} \boldsymbol{\sigma}_{p}}{n_{p s}} \cdot \sum_{s=1}^{n_{p s}} \overline{\nabla S_{i}}\left(\boldsymbol{x}_{p s}\right) .
$$

Since the sub-point algorithm has recently been published [16], we will not discuss it further in the present paper, other than reminding readers that, to ensure energy conservation (to the second order of the spatial and temporal discretization), the velocity gradient at a particle, which is used to calculate stress, also needs to be computed using the new gradient of the shape function as

$$
\overline{\overline{\nabla \widetilde{\boldsymbol{v}}}}\left(\boldsymbol{x}_{p}, t\right)=\sum_{i=1}^{N_{g}} \widetilde{\boldsymbol{v}}_{i}(t) \overline{\overline{\nabla S_{i}}}\left(\boldsymbol{x}_{p}\right)=\sum_{i=1}^{N_{g}} \frac{\widetilde{\boldsymbol{v}}_{i}(t)}{n_{p s}} \sum_{s=1}^{n_{p s}} \overline{\nabla S_{i}}\left(\boldsymbol{x}_{p s}\right) .
$$

\section{Molecular Dynamics Simulation}

In the present work, we use a one-dimensional strong shock propagation in cerium crystal to demonstrate the feasibility of this combination of molecular dynamics simulation and the dual domain material point method. Although the continuum problem is in one dimension, the molecular

dynamics calculation is performed in a three-dimensional periodic domain to minimize the effects of boundary $[3,4,6]$.

In the molecular dynamics calculation, the Embedded Atom Method (EAM) $[25,26]$ potential is used. The total potential energy of a system in EAM method can be written as

$$
E=\frac{1}{2} \sum_{\substack{\alpha, \beta \\ \alpha \neq \beta}} \phi\left(r_{\alpha \beta}\right)+\sum_{\alpha} F\left(\Gamma_{\alpha}\right)
$$


where the first summation is over all atom pairs, $\phi\left(r_{\alpha \beta}\right)$ is the pair potential, $r_{\alpha \beta}$ is the distance between atoms $\alpha$ and $\beta, F$ is the embedding energy as a function of host electron density $\Gamma_{\alpha}$. The host electron density can be written as the sum of the electron densities $\gamma\left(r_{\alpha \beta}\right)$,

$$
\Gamma_{\alpha}=\sum_{\beta(\neq \alpha)} \gamma\left(r_{\alpha \beta}\right)
$$

In this study of cerium metal, a tabulated potential generated by Sheng et al. [27, 28] has been used. The potential data consists of three tables, $F_{\alpha}$ as a function of $\Gamma_{\alpha}, \gamma\left(r_{\alpha \beta}\right)$ and $\phi\left(r_{\alpha \beta}\right)$ as functions of $r_{\alpha \beta}$. If necessary, the data are interpolated using Lagrangian interpolation. By differentiating this energy, we can calculate total force acting on atom $\alpha$ as [29]

$$
\boldsymbol{f}_{\alpha}=-\nabla_{\alpha} E,
$$

where $\nabla_{\alpha}$ denotes the gradient acting on the position of the $\alpha$-th atom.

At the beginning of our calculation, one molecular dynamics (MD) system is generated for each material point. To initialize the MD systems, we first place Cerium atoms in the cubic periodic domains $\left[-L_{p x}^{0} / 2, L_{p x}^{0} / 2\right] \times\left[-L_{p y}^{0} / 2, L_{p y}^{0} / 2\right] \times\left[-L_{p z}^{0} / 2, L_{p z}^{0} / 2\right]$ with $L_{p x}^{0}=L_{p y}^{0}=L_{p z}^{0}$, according to face center cubic (FCC) array. The lattice constant of the arrays is set to the value (5.132 $\AA$ ) where the potential energy $E$ is the minimal. The periodic length is a multiple of this value. To specify initial engineering strain $\varepsilon_{x x}^{e 0}\left(\boldsymbol{x}_{p}\right)$ of the material point in the MD system, for our one-dimensional macroscopic problem, the $x$-coordinate of the atoms and $x$-length $\left(L_{p x}^{0}\right)$ of the periodic domain are multiplied by $1+\varepsilon_{x x}^{e 0}\left(\boldsymbol{x}_{p}\right)$.

To set nonzero initial temperature in the system, the velocities of the atoms are set randomly according to the Maxwell distribution with their magnitudes corresponding to twice the intended initial temperature, and the velocity directions are set according to uniform distribution in the solid angles. The final procedure in the MD system initialization is to relax the system to its thermodynamic equilibrium. In this procedure the equation of motion for atoms are solved as describe below, but with the strain rate set to zero. According to the virial theorem, which is numerically verified, in our cases the total initial kinetic energy is split equally between the kinetic energy and the potential energy among the atoms at the thermodynamic equilibrium; therefore, the relaxed system has the temperature of the intended value. The MD systems are assumed adiabatic in our simulations after this temperature initialization. This initialization of the MD system is 
done once and only once for every material point in the computation. The MD systems are never re-initialized, and their histories are preserved in the present work. The end states of the MD systems of the previous DDMP time step are the initial states for the MD calculations in the next DDMP time step. The only difference in the MD simulations in two consecutive DDMP time steps are the velocity gradients, or the strain rates, used to update the sizes of the periodic computational domains.

With the force $\boldsymbol{f}_{\alpha}$ calculated from (27), we use the leap frog algorithm to advance the MD systems. The half-time step atom velocity is calculated as

$$
\boldsymbol{v}_{\alpha}^{n+1 / 2}=\boldsymbol{v}_{\alpha}^{n-1 / 2}+\frac{\boldsymbol{f}_{\alpha}^{n}}{m_{\alpha}}(\Delta t)_{m d}
$$

where superscripts denote time steps in the MD system, $m_{\alpha}$ is the mass of the atom, and $(\Delta t)_{m d}$ is the time step in the MD simulation. In the very first time step, the half step velocity $\boldsymbol{v}_{\alpha}^{1 / 2}$ is calculated as

$$
\boldsymbol{v}_{\alpha}^{1 / 2}=\boldsymbol{v}_{\alpha}^{0}+\frac{\boldsymbol{f}_{\alpha}^{0}}{2 m_{\alpha}}(\Delta t)_{m d}
$$

The full step velocity $\boldsymbol{v}^{n}$ is calculated as an average of $\boldsymbol{v}_{\alpha}^{n-1 / 2}$ and $\boldsymbol{v}_{\alpha}^{n+1 / 2}$.

In each time step an atom position is first updated as

$$
\boldsymbol{x}_{\alpha}^{*}=\boldsymbol{x}_{\alpha}^{n}+\boldsymbol{v}_{\alpha}^{n+1 / 2}(\Delta t)_{m d}
$$

The communication between the macroscopic system and the molecular dynamics systems is through the strain rate and stress. In every MD time step, using the strain rate $\dot{\varepsilon}_{x x}\left(\boldsymbol{x}_{p}, t\right)$ (which equals to the velocity gradient in this one-dimensional problem) calculated from (24) for this macroscopic time and material point, we update the periodic domain length in the $x$-direction as

$$
L_{p x}^{n+1}=L_{p x}^{n}\left[1+\dot{\varepsilon}_{x x}\left(\boldsymbol{x}_{p}, t\right)(\Delta t)_{m d}\right]
$$

while leaving the periodic domain sizes in the other two dimensions unchanged. To ensure all atoms are within the periodic domain, atoms that have left from one side of the periodic domain are put back through the other side. More precisely, the atom locations are further updated using $\boldsymbol{x}_{\alpha}^{*}$ from (30). For the $x$ coordinate we use

$$
x_{\alpha}^{n+1}=\left(x_{\alpha}^{*}+\frac{L_{p x}^{n+1}}{2}\right) \bmod \left(L_{p x}^{n+1}\right)-\frac{L_{p x}^{n+1}}{2},
$$


and similarly for $y$ and $z$ coordinates. Since there is a velocity gradient in the $x$ direction, if $x_{\alpha}^{n+1} \neq x_{\alpha}^{*}$, then velocity $\left(x_{\alpha}^{n+1}-x_{\alpha}^{*}\right) \dot{\varepsilon}_{x x}\left(\boldsymbol{x}_{p}, t\right)$ is added to the full-step velocity $\boldsymbol{v}_{\alpha}^{n}$ and the halfstep velocity $\boldsymbol{v}_{\alpha}^{n+1 / 2}$ of the atom.

The method presented in this work is intended to resolve the effects of thermodynamic nonequilibrium in macroscopic problems, where the time scale of the macroscopic strain rate is comparable to the thermodynamic relaxation time. We have no choice but to use time steps in the continuum DDMP calculation much smaller than the time scale of the strain rate. Clearly, this method should not be used directly for problems in which the strain rate is small compared to the inverse of the thermodynamic relaxation time. For such systems, for each continuum DDMP time step, we only need to perform sufficient MD time steps for each of the MD systems to reach its local thermodynamic equilibrium [30], then perform a time average and extrapolate the stress to be used in the continuum DDMP time step. In this limit this method reduces to category B of the HMM as described by $[3,4,6,7]$. The only difference is then in the continuum solvers. The finite element and the finite volume methods are used in $[3,4,5]$, while we use the DDMP method, which is more advantageous if the history dependence and large material deformation is of concern.

To calculate stress in the periodic computational domain while avoiding errors related to the interaction pairs near the boundary of our MD domain, we use pair interaction forces and the first line of (16). This is similar to [3], but without the time averaging. As shown in (16), this calculated stress is consistent with virial stress expression of the Cauchy stress, which is independent of force decomposition in the limit of continuum mechanics. In our calculation, the pair interaction force between a pair of atoms is calculated as $[29,31]$

$$
\boldsymbol{f}_{\alpha \beta}=-\left[\frac{\partial \phi\left(r_{\alpha \beta}\right)}{\partial r_{\alpha \beta}}+\frac{\partial F}{\partial \Gamma_{i}} \frac{\partial \gamma}{\partial r_{\alpha \beta}}+\frac{\partial F}{\partial \Gamma_{j}} \frac{\partial \gamma}{\partial r_{\alpha \beta}}\right] \frac{\boldsymbol{r}_{\alpha \beta}}{r_{\alpha \beta}} .
$$

In our simulation the molecular dynamics calculations are performed on a GPU. With this pair interaction force, the calculated stress is passed to CPU, which performs the continuum DDMP calculation as described in the previous section.

To confirm that this calculated stress is in agreement with the classical concept of stress in elasticity theory for continuum mechanics, in Fig. 3 we show stress-strain relation for cerium at initial temperatures of $0 \mathrm{~K}, 300 \mathrm{~K}$, and $600 \mathrm{~K}$. The results are obtained using 256 atoms and 500 atoms in the periodic domain with initial domain sizes of $20.5 \AA$ and $25.7 \AA$. These results are 


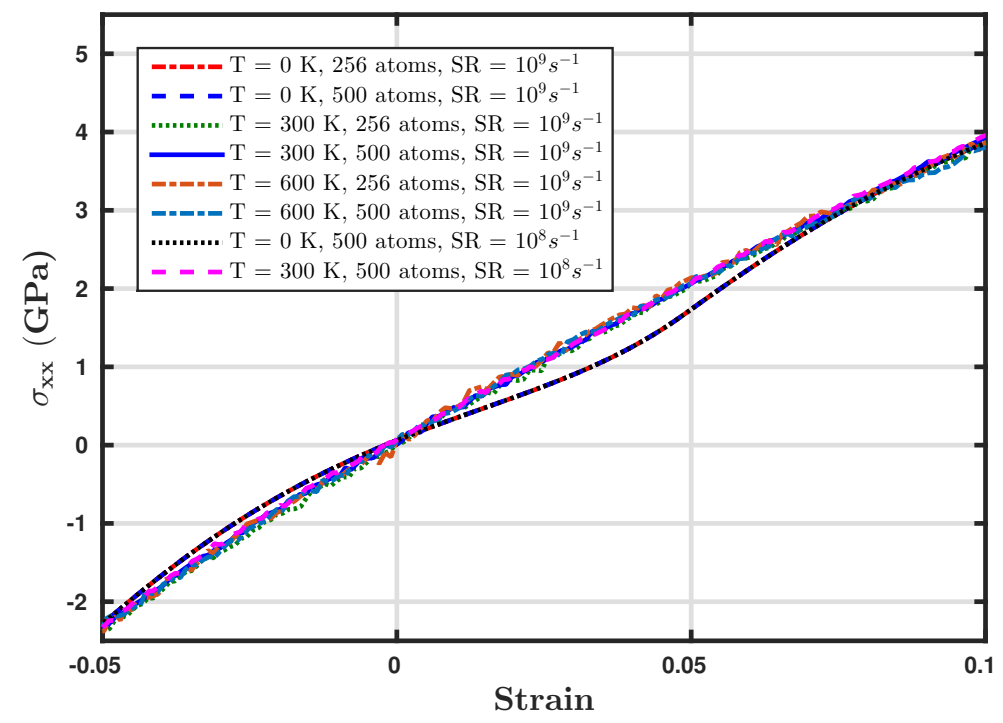

Figure 3: Stress-strain Relation of Cerium at different temperatures. The strain used in this figure is the engineering strain $\varepsilon_{x x}^{e}=\left(L_{p x}^{n}-L_{p x}^{0}\right) / L_{p x}^{0}$.

obtained by imposing strain rates $10^{8} 1 / \mathrm{s}$ and $10^{9} 1 / \mathrm{s}$ to the system using time step $(\Delta t)_{m d}=1$ fs in the MD simulation. Time step $(\Delta t)_{m d}=1 \mathrm{fs}$ is about $1 \%$ of the phonon period for cerium [32]. For cases of nonzero initial temperature, according to the initialization process, at zero strain (undeformed crystal with lattice constant $5.132 \AA$ ), there is a small stress due to thermal fluctuations. The stress plotted in Fig. 3 has been offset by this stress to ensure that the curve passes the origin in the figure. Other than small fluctuations, for a given initial temperature, the stress-strain relation is insensitive to the number of atoms and the strain rates used in our MD simulations. The Young's moduli can be calculated from the slopes of the curves at zero strain in this plot and are found to be $16.9 \mathrm{GPa}$ at temperature $0 \mathrm{~K}$ and $25.6 \mathrm{GPa}$ at temperature $300 \mathrm{~K}$, compared to the value of $25.3 \mathrm{GPa}$ at $300 \mathrm{~K}$ obtained by Sheng et al. [27, 28].

\section{Numerical Results and Discussion}

In this section we explore the numerical properties of the multiscale calculation described above. Since the main purpose of this work is to study a numerical method to perform multiscale calculations, instead of improving the MD method or the EAM potential used in our calculations, we compare our results from our multiscale simulations to those obtained from the direct MD simula- 
tions, rather than to experimental values. We study a one-dimensional cerium bar of length about $500 \mathrm{~nm}$. Although the multiscale method described in the present work can be used to compute macroscopic problems, we limit our comparison with the MD results to this small length because of length limit of the MD simulations. Initially, the left half of the bar is compressed to a specified engineering strain and held. In the combined MD-DDMP simulation, after proper initialization of densities on mesh nodes, the MD systems for the material points are initialized with the initial strain and temperature as described in the last section. Initialization of the direct MD simulation is similar. Atoms are placed in the computational domain according to FCC array. In the direct MD calculation, we use periodic boundary condition in $y$ and $z$ directions and reflective boundary condition on both ends of the cerium bar. There are 12 lattices in the $y$ and $z$ directions and 1200 lattices in the $x$ direction. To specify an initial engineering strain $\varepsilon_{x x}^{e 0}$ in left half of the bar, $x$-distance between atoms on the left is adjusted by a factor of $1+\varepsilon_{x x}^{e 0}$.

At the time $t=0$, the hold is released causing a compression shock to propagate to the right and a rarefaction wave to propagate to the left. In the direct MD simulation, the equations of motion for the atoms are solved as described in the last section. To calculate the stress from this direct MD simulation, the entire MD domain is divided into 100 sections along the $x$-direction. We treat each section as a representative volume, and use the first line of (16) to calculate the stress in this section.

For DDMP and combined DDMP-MD calculations, the computational domain consists of 400 cells for the results shown in Figs. 4 to 9. To avoid difficulty associated with the initial discontinuity, as in many particle based methods [33, 34], we use a hyperbolic tangent function to smooth the initial engineering strain across 26 cells, with 13 cells on each side of the initial discontinuity. As shown in Fig. 4 in the case of weak shock, the shock front is broadened to about 50 cells for both the continuum DDMP and the multiscale MD-DDMP calculations due to artificial viscosity. In this work the stress from the artificial viscosity only involves the linear term $-0.5 \rho c_{s}|\Delta v|[35]$, and is applied only if the strain rate is negative. Using the same artificial viscosity for strong shocks, shock front is only broadened by 4 cells to total of 17 cells for the multiscale MD-DDMP calculation and is broadened by 29 cells to total about 42 cells for the continuum DDMP calculations as shown in Figs. 5 to 10.

Figure 4 shows the comparison among the results from the full MD simulation using about 


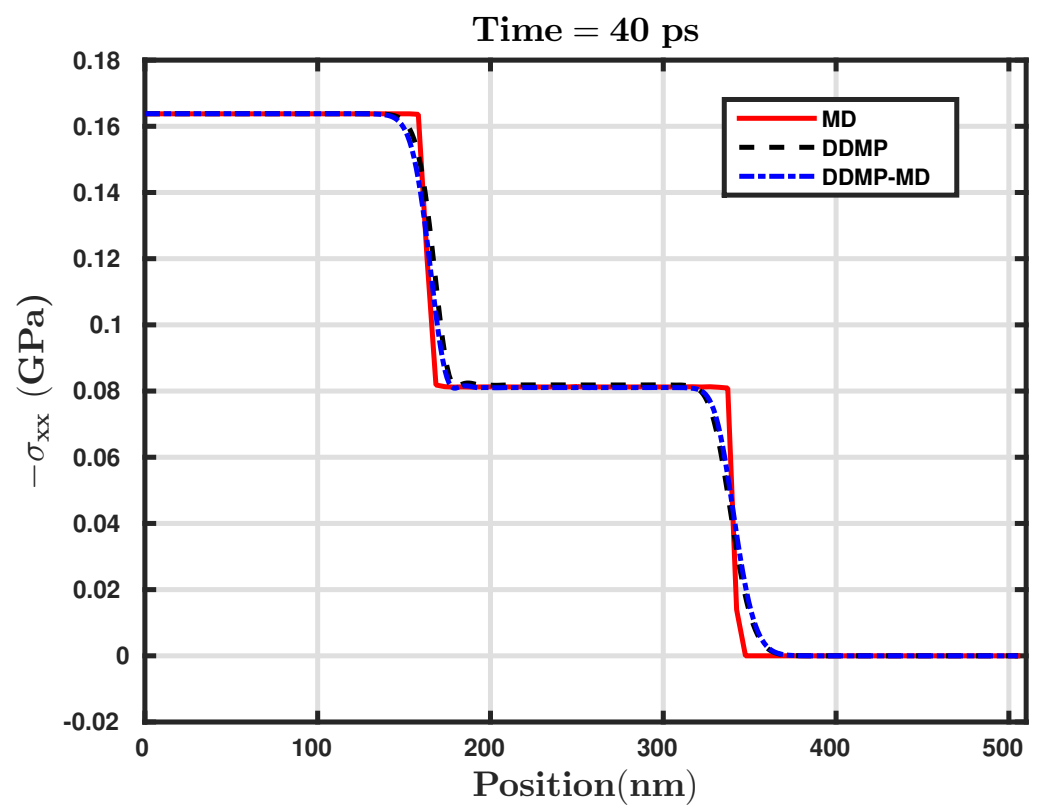

Figure 4: Shock wave propagation for weak shock at $0 \mathrm{~K}$ initial temperature calculated using 400 cells with 1 material point per cell and 8 sub-points per material point.

700,000 atoms, the continuum DDMP calculation using 400 equal cells with initially 1 material point per cell and 8 sub-points per material point [16], and the multiscale DDMP-MD simulation with the same numbers of cells and material points as in the DDMP calculation. For this case of moderate initial compressive strain of $0.5 \%$, corresponding to about $0.164 \mathrm{GPa}$ compressive stress on the left, although the combined DDMP-MD simulation result is slightly closer to the MD calculation, the overall results are in good agreement with each other.

Using the same numerical arrangement, we calculated a case with ten times higher initial compressive strain $5.0 \%$ on the left of the bar. The results are shown in Fig. 5. In this figure, the continuum DDMP results deviate from the MD results showing smaller wave speeds for both the compression and rarefaction waves. The deviation is not caused by the DDMP numerical method, but rather by the constitutive assumption about the linear elasticity of the material. The multiscale DDMP-MD results are much closer to the MD results, especially in the center region of the figure. In this region, the material is significantly perturbed by the compression or rarefaction wave, and is not in the thermodynamic equilibrium. Such thermodynamic nonequilibrium is not considered in the linear elastic model used in the DDMP calculation, resulting the difference between the DDMP and MD results. The effect of this thermodynamic nonequilibrium is captured in the MD 


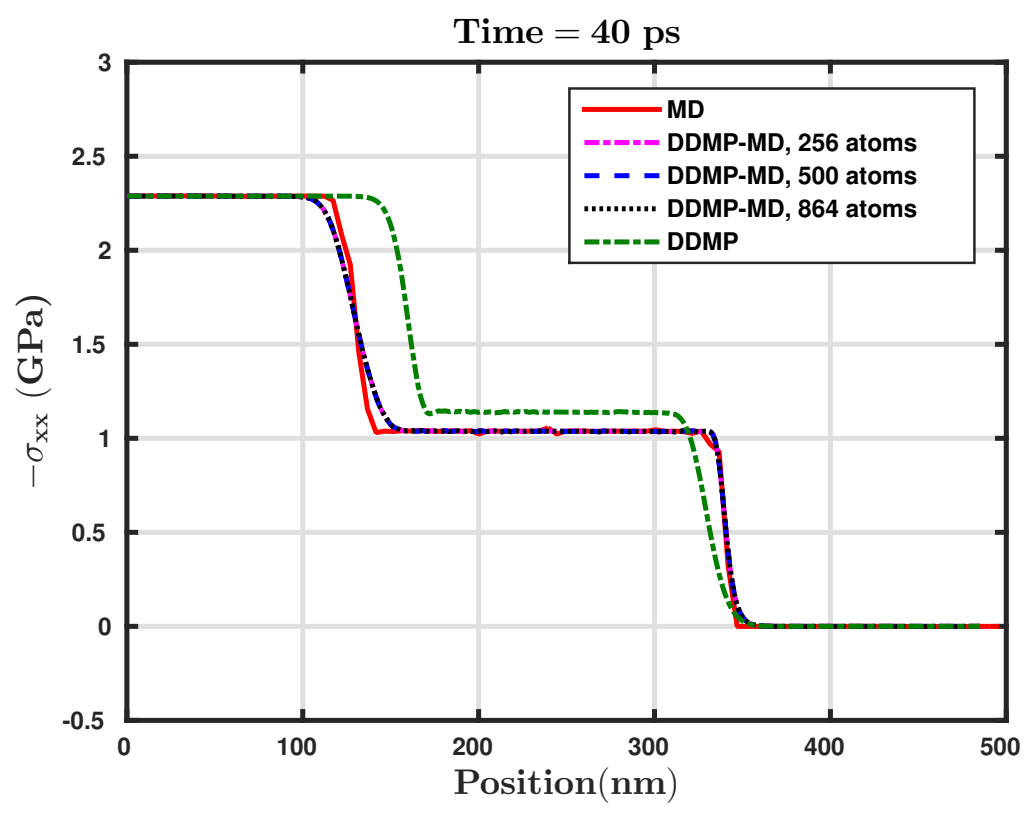

Figure 5: Propagation of strong shock at $0 \mathrm{~K}$ initial temperature calculated using 400 cells with 1 material point per cell and 8 sub-points per material point.

simulation performed at each material point leading to good agreement between the results from the MD and the DDMP-MD combined multiscale calculations. In this figure, we also show the combined DDMP-MD results obtained from different number of atoms in a MD system. There are only slight differences between the results by using different number of atoms.

Figure 6 shows similar calculation for a case with $300 \mathrm{~K}$ initial temperature. In this figure, again the multiscale DDMP-MD result is very close to that from the MD simulation. In this figure the comparison between the continuum DDMP result and the MD results is much better than in Fig. 5, because compared to the system starting from zero absolute temperature, the system with a finite temperature relaxes faster to its thermodynamic equilibrium, where the continuum theory provides a good approximation. This is also the reason for good agreement among all the results in Fig. 4 since the system was never far away from thermodynamic equilibrium for the weak shock. Figure 6 also shows noise in the stresses obtained from both the direct MD and DDMP-MD combined calculations. This noise can be reduced by using larger number of atoms in both calculations. The result obtained using 500 atoms per material point is smoother than that obtained using 256 atoms per material point. These results show that even with a small number (256 or 500 ) of atoms in our MD simulation for each material point, the results still capture the significant effects of 


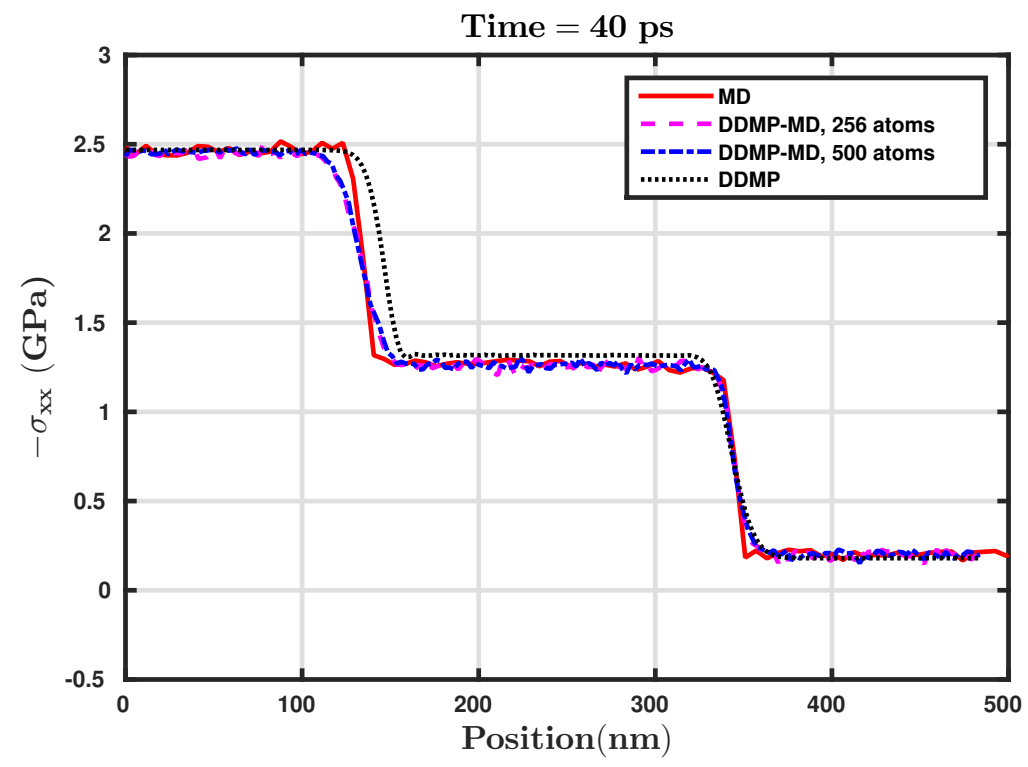

Figure 6: Propagation of strong shock at $300 \mathrm{~K}$ initial temperature calculated using 400 cells with 1 material point per cell and 8 sub-points per material point. In the combined DDMP-MD results, stresses at material points are obtained from the MD simulations using 256 and 500 atoms per material point.

thermodynamic nonequilibrium with reasonable accuracy. In this calculation, for the unperturbed material on the right, the initial stress is negative because of velocity fluctuations of the atoms as represented by the first term in (16) for this case of finite temperature.

In Figs. 4, 5 and 6, the entire domain MD results are obtained from calculations performed in parallel on a NVIDIA Tesla K20 GPU. Each took about 6 hours. The MD calculations for the multiscale DDMP-MD method are also performed on the GPU, whereas the continuum scale calculations are performed in a CPU. The DDMP-MD calculations took about 15 minutes each, and the continuum DDMP calculation took only a few CPU seconds. We use time steps of 500 fs in the DDMP calculations, while the time step for the MD simulation is $1 \mathrm{fs}$. The 500 fs time step in the continuum part of the simulation corresponds Courant number about 0.8, based on the wave speed, which varies during the calculation. For the combined DDMP-MD calculation, the stress used in the continuum DDMP calculation is calculated using (16) at the last MD time step immediately before the continuum time step. As another option one can also use the average stress calculated at each MD time step. The difference shown in Fig. 7 is not significant, but the 


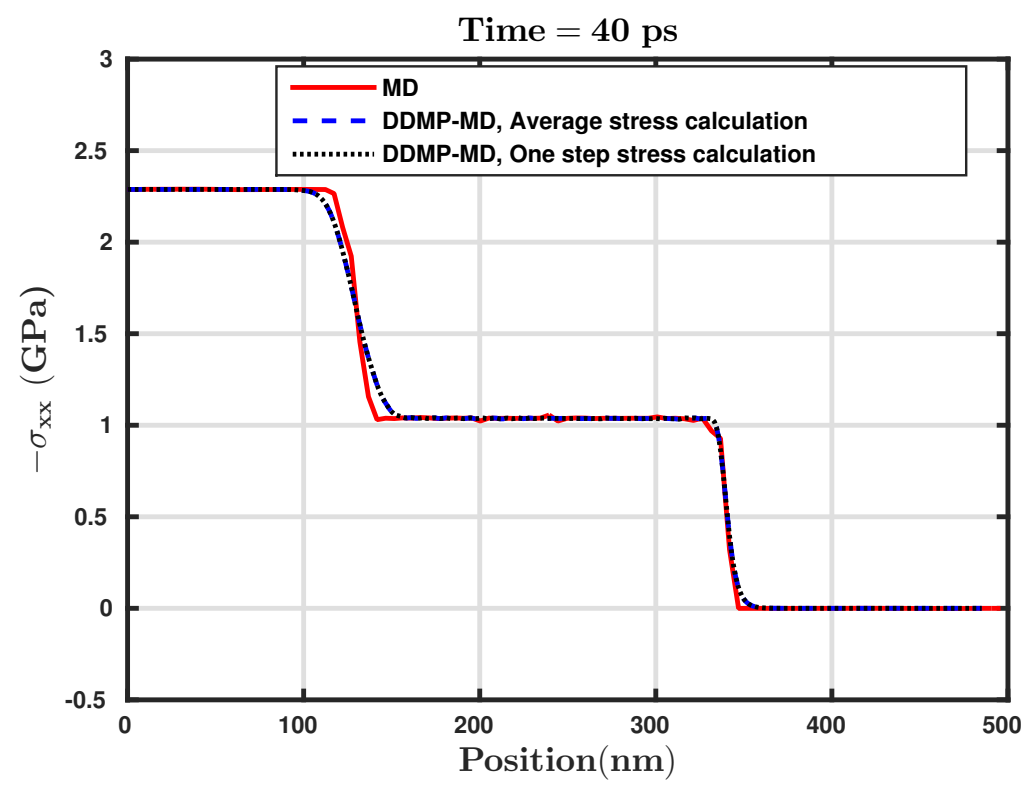

Figure 7: Comparison of results from using the stress calculated from one MD time step stress and using the average stress over the one DDMP time step for cases of $0 \mathrm{~K}$ initial temperature.

computation cost differs remarkably. Since the stress calculation requires global sum over the atom pairs, which cannot be performed effectively in parallel on a GPU, the calculation using the average stress is about 1.8 times slower than the method that simply evaluates the stress at the last time step of the MD simulation before the DDMP calculation. For this reason, the latter method is used to obtain all other results reported in the present work.

To study numerical properties of the combined DDMP-MD simulation, without the contamination of the noise from thermal fluctuations, in the following, we use systems with initial temperature of absolute zero to study the effect of artificial viscosity, the effect of the number of material points, and mesh sizes used in the calculation. The effect of artificial viscosity is shown in Fig. 8. Artificial viscosity is needed to suppress the Gibbs phenomenon near the compression wave front.

In DDMP calculation, we place a number of material points in cells evenly at the beginning of a calculation. The results shown in Fig. 9 are obtained with different number of material points in such placement. Because the material points are Lagrangian, they move with the material leading to increase or decrease of the number of material points in the cell during the calculation. The number of material points per cell in the figure only refers to the initial placement of the points. In the figure, we compare the results obtained by initially placing 1 material point per cell to that 


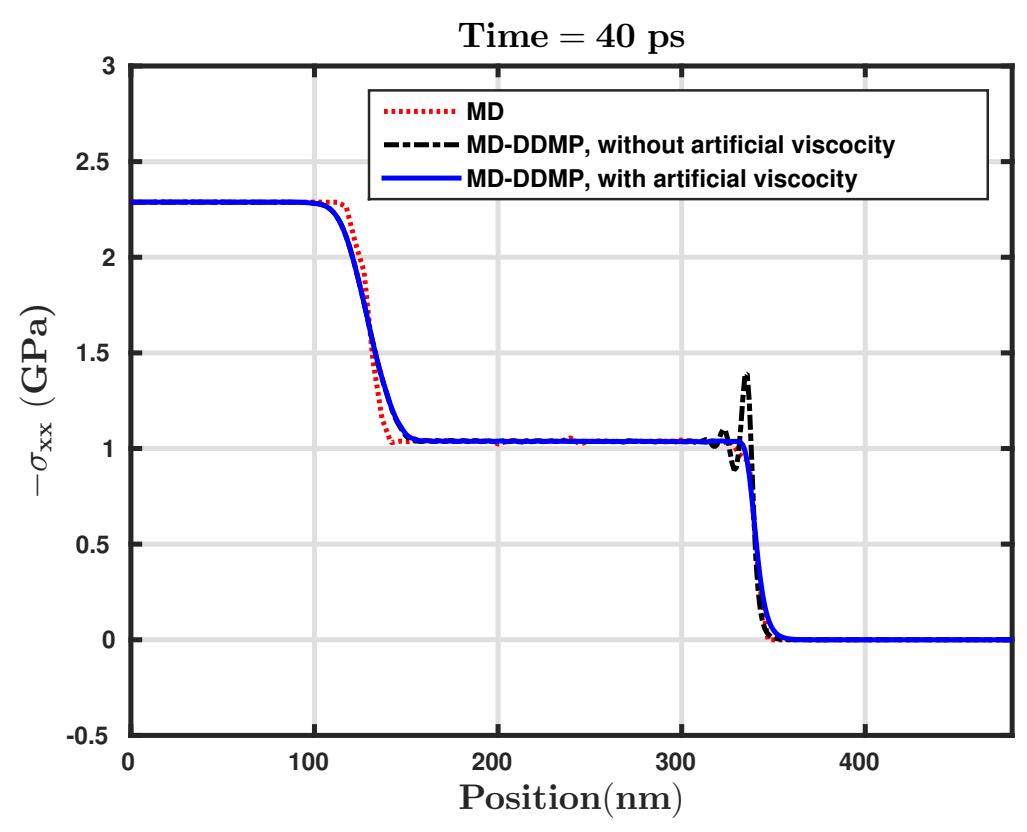

Figure 8: Effects of artificial viscosity for cases of $0 \mathrm{~K}$ initial temperature.

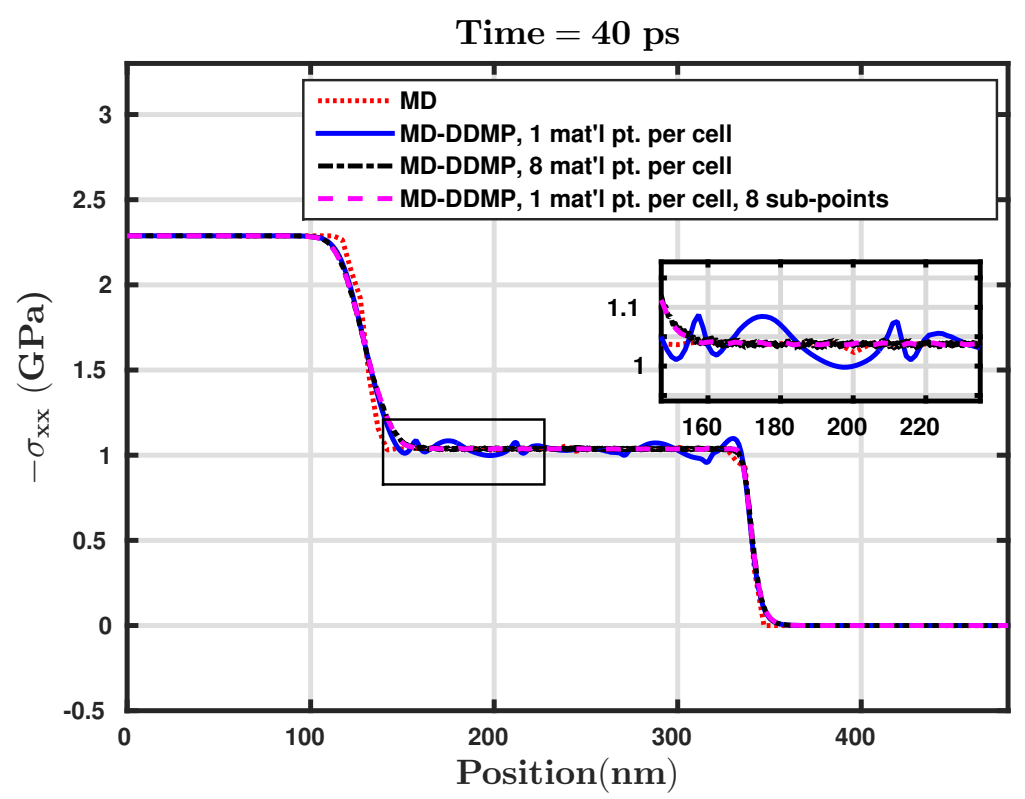

Figure 9: Effects of number of material points or sub-points for cases of $0 \mathrm{~K}$ initial temperature. 
obtained by initially placing 8 material points per cell for the case of strong shock. The result from using 1 material point per cell is noisier as can be seen in the sub figure. The noise is a result of low accuracy of the numerical integration scheme used in the material point method. By using the sub-point algorithm [16], the noise can be significantly reduced. In Fig. 9, we also plot the result obtained using 1 material point per cell and 8 sub-points per material point. This result is barely different from the result obtained using 8 material points per cell. The CPU times are about 15 minutes for the two calculations using 1 material point per cell with or without using the sub-points. These results show that the use of sub-points leads to a significant speedup compare to the time needed for calculation using 8 material points per cell, which took about 110 minutes, without sacrificing numerical accuracy. The use of sub-points is especially advantageous in this multiscale calculation, where stress evaluation at the material points is expensive.

In Fig. 10, we show effects of cell size on this multiscale calculation. This shows the results converge to the correct solution as mesh is refined. The results of the shock propagation calculated in this work are in a very small $(500 \mathrm{~nm})$ domain. This small domain is chosen because we want to compare our multiscale calculation with the MD calculation. While the MD calculation is restricted to a small domain, the combined DDMP-MD simulation is not because there is no difference in the stress calculation method when this combined DDMP-MD is used for much larger domains. It can be used to simulate thermodynamic nonequilibrium phenomena involving large spatial domains instead of limited to some special areas, such as phase interfaces or crack tips $[36,37,38]$. To further develop this method and to consider effect of crystal defects, such as twinning and dislocation, large MD systems for the material points are needed.

The size of time steps (500 fs) in the continuum calculation used in the present paper is quite small for a typical continuum scale calculation. If, in the systems of interest, the effect of thermodynamic nonequilibrium is important, physical interactions at this small time scale have to be resolved to capture the nonequilibrium effects. Fortunately, for most macroscopic problems, the system relaxes to a thermodynamically equilibrium state quickly compared to the macroscopic time scale. After that time if the constitutive relation or the equation of state for the material is available, this multiscale method is no longer needed. In cases where the constitutive relation is not available even in the thermodynamically equilibrium state, one can explore the time extrapolation and adaptive spacetime methods $[3,6,7]$ by only performing this multiscale simulation in selected 


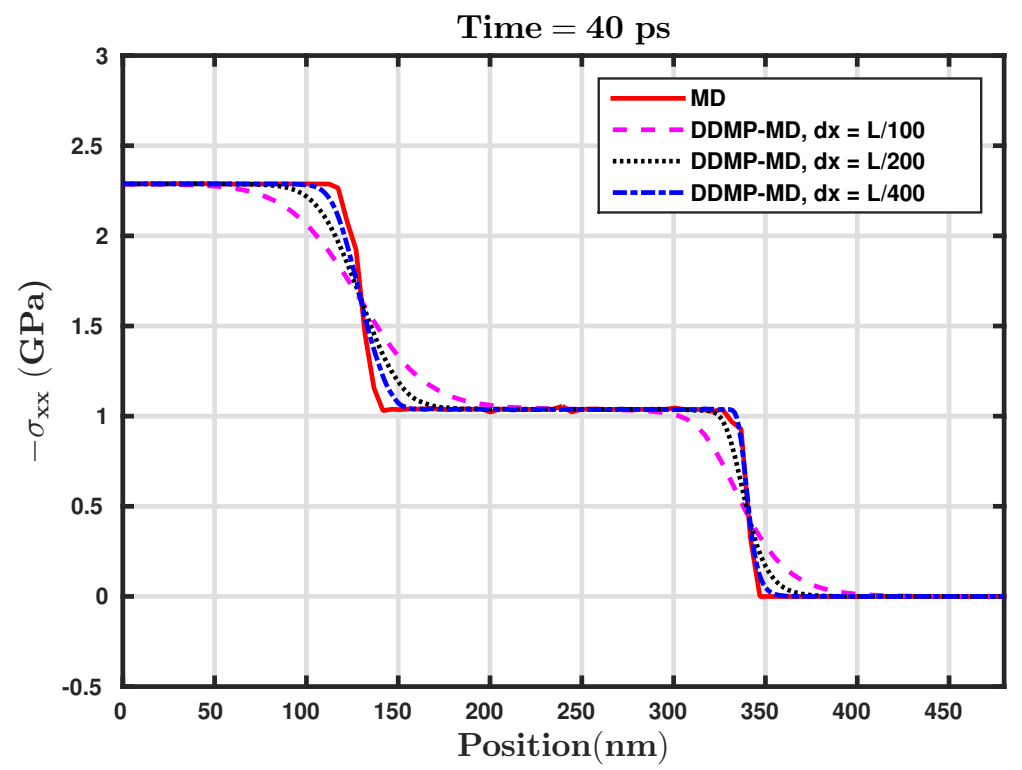

Figure 10: Effect of mesh size in shock propagation at $0 \mathrm{~K}$ initial temperature calculated with 1 material point per cell and 8 sub-points per material point.

time intervals and then using the extrapolated closure quantities to advance the macroscopic states.

\section{Concluding Remarks}

By taking advantages of the unnecessity of communication among the material points, an efficient multiscale simulation method is developed based on the dual domain material point (DDMP) method. In this method, the stresses on each material point are calculated independently without a need to communicate with other material points. The material points only communicate with mesh nodes. For problems, where the traditional constitutive relations are not available, we perform molecular dynamics (MD) simulation to obtain the stress and use it in the continuum scale DDMP calculation. Because no communication among material points is required, in this method, the MD simulations are performed independently and in parallel on a GPU.

The choice of the DDMP method is based on its capability of tracking material deformation history for cases involving extreme material deformations, which are often accompanied by high strain rates, where the assumption of thermodynamic equilibrium becomes invalid, and history dependence becomes important. In the DDMP method, especially with the recent enhancement using sub-points, the noise caused by material points moving across cell boundaries is suppressed, 
and highly accurate results can be obtained.

Compared to other multiscale methods, this method has an advantage in its capability of simulation a macroscopic domain because each material point is treated as a representative piece of the material that moves and deforms with the material, and carries the history with it.

Although for complex materials, a large scale MD simulation for each material point is needed to capture effects such as crystal defects and dislocations, our examples show that the effect of thermodynamic nonequilibrium is well captured. The results reported in the present work are limited to one-dimensional shocks. The formulations presented in the paper are general. Although we believe its extension to multi-dimensional problems and other loading conditions is rather straightforward because the DDMP method has been implemented to three-dimensional calculations with complex deformation, the efficiency of the combined multiscale calculation still needs to be explored, and the consistency conditions for the communication schemes between MD scale and continuum scale calculations have yet to be studied.

\section{Acknowledgments}

The authors would like to acknowledge many useful and in-depth discussions with Dr. Rick M. Rauenzahn of Los Alamos. This work was performed under the auspices of the United States Department of Energy. The Stockpile Safety and Surety Program, the Joint DoD/DOE Munitions Technology Development Program, and the ASC Program provided the financial support for this work.

\section{References}

[1] J. H. Irving, I. G. Kirkwood, The statistical theory of transport process, iv. the equations of hydrodynamics, J. Chem. Phys. 18 (1950) 817-829.

[2] D. Z. Zhang, X. Ma, R. Rauenzahn, Interspecies stress in momentum equations for dense binary particulate systems, Phys. Rev. Lett. 97 (4) (2006) 048301.

[3] X. Li, W. E, Multiscale modeling of the dynamics of solids at finite temperature, Journal of the Mechanics and Physics of Solids 53 (2005) 1650 - 1685. 
[4] W. Ren, W. E, Heterogeneous multiscale method for the modeling of complex fluids and micro-fluidics, Journal of Computational Physics 204 (2005) 1-26.

[5] X. Li, J. Z. Yang, W. E, A multiscale coupling method for the modeling of dynamics of solids with application to brittle cracks, Journal of Computational Physics 229 (2010) 3970 - 3987.

[6] A. Abdulle, W. E, B. Engquist, E. Vanden-Eijnden, The heterogeneous multiscale method, Acta Numerica (2012) 1-87.

[7] B. Kraczek, S. Miller, R. Haber, D. Johnson, Adaptive spacetime method using riemann jump conditions for coupled atomistic-continuum dynamics, Journal of Computational Physics 229 (2010) $2061-2092$.

[8] M. Jemison, M. Sussman, M. Shashkov, A coupled level set and volume-of-fluid method for computing 3d and axisymmetric incompressible two-phase flows, Journal of Computational Physics 162 (2000) $301-337$.

[9] M. Jemison, M. Sussman, M. Shashkov, Filament capturing with the multimaterial momentof-fluid method, Journal of Computational Physics 285 (2015) 149 - 172.

[10] D. Z. Zhang, C. Liu, F. H. Harlow, Effects of nonuniform segment deformation on the constitutive relation of polymeric solids, Physical Review E 66 (2002) 051806 (1-15).

[11] D. Z. Zhang, R. M. Rauenzahn, A viscoelastic model for dense granular flows, J. Rheol. 41 (6) (1997) 1275-1298.

[12] N. C. Admal, E. B. Tadmor, A unified interpretation of stress in molecular systems, Journal of Elasticity 100 (2010) $63-143$.

[13] N. C. Admal, E. B. Tadmor, The non-uniqueness of the atomistic stress tensor and its relationship to the generalized beltrami representation, Journal of the mechanics and physics of solids 93 (2016) $72-92$.

[14] L. E. Malvern, Introduction to the mechanics of a continuous medium, Prentice-Hall, Upper Saddle River, 1969. 
[15] D. Z. Zhang, X. Ma, P. Giguere, Material point method enhanced by modified gradient of shape function, Journal of Computational Physics (2011) doi:10.1016/j.jcp.2011.04.032.

[16] T. R. Dhakal, D. Z. Zhang, Material point methods applied to one-dimensional shock waves and dual domain material point method with sub-points, Journal of Computational Physics 325 (2016) 301- 313.

[17] D. Sulsky, Z. Chen, H. L. Schreyer, A particle method for history-dependent materials, Computational Methods in Applied Mechanics and Engineering 118 (1994) 179-196.

[18] B. Jayaraman, X. Ma, P. T. Giguere, D. Z. Zhang, Single-and multi-velocity formulations for impact and pulverization, International Journal of Impact Engineering 49 (2012) 11-21.

[19] P. G. Ciarlet, The Finite Element Method for Elliptic Problems, North-Holland Publishing Company, Amsterdam, 1978.

[20] X. Ma, D. Z. Zhang, P. T. Giguere, C. Liu, Axisymmetric computation of Taylor cylinder impacts of ductile and brittle materials using original and dual domain material point methods, International Journal of Impact Engineering 54 (2013) 96-104.

[21] D. Z. Zhang, B. Jayaraman, Equations and closure models for material pulverization and debris flow, International Journal of Multiphase Flow 56 (2013) 149-159.

[22] C. C. Long, D. Z. Zhang, C. A. Bronkhorst, G. T. Gray, Representing ductile damage with the dual domain material point method, Comput. Methods Appl. Mech. Engrg. 300 (2016) 611 627.

[23] J. U. Brackbill, H. M. Ruppell, FLIP: a method for adaptively zoned, particle-in-cell calculations of fluid flows in two dimensions, Journal of Computational Physics 65 (1986) 314-343.

[24] F. H. Harlow, The particle-in-cell computing method for fluid dynamics, Methods Comput. Phys 3 (1964) 319.

[25] M. S. Daw, M. I. Baskes, Embedded-atom method: Derivation and application to impurities, surfaces, and other defects in metals, Phys. Rev. B 29 (1984) 6443-6453. 
[26] S. M. Foiles, M. I. Baskes, M. S. Daw, Embedded-atom-method functions for the fcc metals cu, ag, au, ni, pd, pt, and their alloys, Phys. Rev. B 33 (1986) 7983-7991.

[27] H. W. Sheng, M. J. Kramer, A. Cadien, T. Fujita, M. W. Chen, Highly optimized embeddedatom-method potentials for fourteen fcc metals, Phys. Rev. B 83 (2011) 134118.

[28] A. Cadien, Q. Y. Hu, Y. Meng, Y. Q. Cheng, M. W. Chen, J. F. Shu, H. K. Mao, H. W. Sheng, First-order liquid-liquid phase transition in cerium, Phys. Rev. Lett 110 (2013) 125503.

[29] Y. Mishin, Handbook of Materials Modeling, ed. S. Yip, Springer, 2005.

[30] R. Luzzi, A. Vasconcellos, On the nonequilibrium statistical operator method, Fortsclir. Phya. 38 (1990) 887-922.

[31] X. Wu, X. Li, On consistent definitions of momentum and energy fluxes for molecular dynamics models with multi-body interatomic potentials, Modelling and Simulation in Materials Science and Engineering 23 (2015) 015003.

[32] J. Fu, J. Zhao, Gupta potential for rare earth elements of the fcc phase: lanthanum and cerium, Modelling Simul. Mater. Sci. Eng. 21 (2013) 065003.

[33] J. J. Monaghan, SPH and Riemann solvers, Journal of Computational Physics 136 (1997) 298 $-307$.

[34] L. D. G. Sigalotti, H. López, A. Donoso, E. Sira, J. Klapp, A shock-capturing sph scheme based on adaptive kernel estimation, Journal of Computational Physics 212 (2005) 124 - 149.

[35] E. J. Caramana, M. J. Shashkov, P. P. Whalen, Formulations of artificial viscosity for multidimensional shock wave computations, Journal of Computational Physics 144 (1998) 70 - 97.

[36] Y. Lee, C. Basaran, A multiscale modeling technique for bridging molecular dynamics with finite element method, Journal of Computational Physics 253 (2013) 68 - 85.

[37] E. Saether, V. Yamakov, E. H. G. 1, An embedded statistical method for coupling molecular dynamics and finite element analyses, Int. J. Numer. Meth. Engng 78 (2009) 1292 - 1319.

[38] K. Gu, C. B. Watkins, J. Koplik, Atomistic hybrid dsmc/nemd method for nonequilibrium multiscale simulations, Journal of Computational Physics 229 (2010) 1381 - 1400. 